\title{
Diagnóstico social, demográfico y económico, y situación de pobreza del municipio de Guamal, departamento del Magdalena, Colombia*
}

\author{
Social, demographic and economic diagnosis, and poverty \\ situation of the municipality of Guamal, Magdalena, Colombia
}

\author{
Marta Elena Correa ${ }^{1}$ \\ Natalia Gómez Montoya ${ }^{2}$ \\ Raúl Antonio Mejía Contreras ${ }^{3}$
}

\section{Resumen}

Este artículo presenta las características sociodemográficas y económicas del municipio de Guamal, Magdalena, que describen la pobreza, y lo compara con la condición general del sector rural colombiano, fue realizado a partir de una investigación descriptiva exploratoria, utilizando la información de la base de datos del SISBEN, a la que se le aplicaron criterios de estadística descriptiva. Además, con esta misma información se elaboró una matriz de doble entrada (la cual cruza el perfil habitacional con áreas o zonas del municipio) útil para efectos de planificación. Por último, se realizó un proceso de análisis y reflexión participativa de la misma población a través de grupos focales, que arrojó resultados acerca de la percepción de la comunidad frente a sus condiciones de vida, encontrando deficiencias básicas en ellas en materia de cobertura y calidad de salud, educación superior, vivienda, saneamiento básico, participación social, confianza y convivencia familiar, situaciones todas estas más graves en la zona rural del municipio donde habita el $70 \%$ de la población, lo que configura una situación de pobreza que es un obstáculo para la integración social y es, a la vez, generadora de conflictos y desestabilizadora de las instituciones democráticas, lo cual desencadena consecuencias negativas en la sociedad en su conjunto.

\section{Palabras clave}

Pobreza, caracterización socioeconómica, demográfica, perfil habitacional, saneamiento básico.

Códigos de clasificación JEL: J11, Q26, R14, R52

* Artículo derivado de la investigación "Diagnóstico social, demográfico y económico, y situación de pobreza del municipio de Guamal, departamento del Magdalena, Colombia”. Auspiciado por la Universidad Pontificia Bolivariana y la Congregación de los Siervos Misioneros de la Santísima Trinidad.

1 Magíster en Desarrollo, docente investigadora Facultad de Trabajo Social Universidad Pontificia Bolivariana Correo electrónico: marta.correa@upb.edu.co; martaelena.correa@gmail.com

2 Estudiante de Trabajo Social, Facultad de Trabajo Social, Universidad Pontificia Bolivariana. Correo electrónico: natalia.gomezmon@upb.edu.co

3 Trabajador social, Facultad de Trabajo Social Universidad Pontificia Bolivariana. Correo electrónico. raul.mejiaco@upb.edu.co 


\begin{abstract}
This paper presents the social,demographic and economic characteristics of Guamal (Magdalena) that describe the poverty in this municipality and compares it with the general condition of the rural colombian sector, based on the descriptive and exploratory research carried out using information from the SISBEN database, which was applied criteria of descriptive statistics. In addition, with this same information, a matrix of double entry (which crosses the habitational profile with areas of the municipality) was elaborated for planning purposes. Finally, a process of analysis and participatory reflection of the same population was carried out through focus groups, which showed results about the community's perception of their living conditions, finding basic deficiencies in coverage and quality of health, higher education, housing, basic sanitation, social participation, trust and coexistence family, situations are all more serious in the rural area of the municipality where $70 \%$ of the population lives, which constitutes a situation of poverty that is an obstacle to social integration and is both a source of conflict and destabilizing of democratic institutions, which has negative consequences for all the society.
\end{abstract}

\title{
Keywords
}

Social, demographic and economic characterization, , habitational profile, basic sanitation, poverty.

\section{Introducción}

El departamento del Magdalena tiene un gran potencial de desarrollo, debido a la riqueza de sus recursos naturales y al capital cultural de sus pueblos, sin embargo, es uno de los departamentos más rezagados del país, con los mayores índices de pobreza, desigualdad y miseria, esta situación es corroborada a través de los distintos estudios socioeconómicos revisados.

El Magdalena es uno de los departamento más agrícolas de Colombia y de la región Caribe en su conjunto (Romero, 2006). El Departamento Nacional de Estadística - DANE en el Censo del 2005 muestra cifras del departamento del Magdalena que señalan un alto índice de pobreza. El NBI es del 47,68; en miseria 22.9\%; con deficiencias en la calidad de la vivienda $22.8 \%$; personas en hacinamiento $19.41 \%$; con carencia de servicios 16,19\%; inasistencia al colegio 5,66\%. Cifras que ubican al departamento en una línea de pobreza por encima de la media del país, donde el índice de necesidades básicas insatisfechas (NBI) es de 27,7\% y de miseria de 10,6\%. En cuanto al indicador de pobreza extrema para dicho departamento en 2013 fue de $15,6 \%$ y el coeficiente Gini ${ }^{4}$ fue de $0,496 \%$ (DANE, 2014).

A esta situación se suma el flagelo de un conflicto armado que ha agobiado al país durante los últimos 50 años, la débil presencia del Estado y los altos índices de corrupción pública que han marcado la historia de Colombia (Observatorio Nacional de Paz, 2010).

El presente artículo tiene como objetivo caracterizar y visibilizar las condiciones sociodemográficas y económicas del municipio de Guamal en el Magdalena, en lo relativo a la insatisfacción de sus necesidades básicas, para conocer su condición de pobreza, y señalar algunas alternativas de inversión social que sean posibles de rea-

4 El Coeficiente Gini en economía es un método que mide el grado de desigualdad en la distribución de los ingresos o también la desigualdad de la riqueza, escala de medida va de 0 a 1 , donde a cerca del 0 , tiende a la equidad y por el contrario, cerca de 1, mayor desigualdad. 
lizar mediante cooperación entre los entes públicos y privados, con la participación de la población del municipio de Guamal.

\section{Metodología}

Para la realización de este estudio se llevó a cabo una revisión de fuentes secundarias "consistente en la selección y recopilación de información por medio de la lectura y crítica de documentos y materiales bibliográficos, de bibliotecas, hemerotecas, centros de documentación e información" (Baena, 1988, p. 72).

Dentro de las fuentes secundarias se consideraron estudios, como El perfil social del municipio en el Censo del 2005; Clasificación del Sisbén; el Plan de Desarrollo del municipio; Encuestas de calidad de vida y de necesidades básicas insatisfechas; Resultados de Pobreza y Desigualdad del DANE, y otras investigaciones que posibilitaron el acercamiento y reconocimiento de las problemáticas socioeconómicas de la población estudiada.

Posteriormente, se realizó una caracterización del municipio apoyada en una metodología mixta, a partir de la organización y consolidación de datos estadísticos tomados de la base de datos del Sisbén, y a través de la elaboración de una matriz de doble entrada ${ }^{5}$ (que cruza información sobre el perfil habitacional y las áreas o zonas del municipio) para el procesamiento del conjunto de información del Sisbén sobre los corregimientos y la cabecera municipal de Guamal. De esta manera, se establecieron los índices de hacinamiento y saneamiento básico, los cuales se representaron gráficamente utilizando una herramienta que se ha denominado semáforo que se constituye en un instrumento indicativo para efectos de planeación. Este instrumento presenta color rojo para las situaciones críticas que requieren una prioritaria intervención, con color amarillo las situaciones intermedias que generan alerta y en verde las situaciones que permiten un plazo más amplio en la intervención, denominadas aceptables.

Se realizó un proceso de análisis y reflexión participativa de la misma población, mediante la aplicación de la técnica de grupos focales, con la cual se obtuvo y registró la autopercepción de la misma población. Se hicieron 5 entrevistas con líderes formales e informales del municipio, con el fin de favorecer un análisis participativo que sirviera para conocer y reflexionar acerca de las carencias en la satisfacción de sus necesidades que la población considera más apremiantes.

El enfoque de la caracterización socioeconómica es descriptivo-exploratorio, porque con él se pretendió observar, detallar e interpretar las características socioeconó-

$5 \quad$ Esta matriz de doble entrada siguió los lineamientos generales recomendados en la metodología elaborada para Corantioquia (2003) en el Convenio Interadministrativo No. 4300 de 2003 con la Universidad Nacional de Colombia. Al respecto, agradecemos la asesoría y el apoyo brindado por el magíster Jhon Jaime Bustamante, profesor-investigador titular de la Escuela de Ciencias Sociales de la Universidad Pontificia Bolivariana. 
micas de la población de Guamal. El diseño de este estudio fue de corte transversal, debido a que los datos se recolectaron en un período de tiempo determinado (un mes).

\section{Marco teórico}

Este trabajo se realizó a partir del concepto de diagnóstico social de Escalada y Fernández (2004), quienes plantean:

La investigación diagnóstica supone análisis de situaciones. El análisis de la situación es un momento complejo que nos desafía a conocer lo que está sucediendo en una determinada representación de la realidad que denominamos situación, incluyendo lo que está sucediendo a quienes están actuando sobre y dentro de los límites de esa realidad. La investigación diagnóstica como momento privilegiado de articulación entre conocimiento e intervención contiene en su definición las complejas relaciones entre sujetos (actores), prácticas (acción) y escenario (estructura) (p. 76).

Escalada y Fernández (2004) hacen énfasis en que la investigación diagnóstica es un tipo específico de investigación empírica, que ayuda a comprender los hechos sociales "situados" o "contextuados", brindando un doble resultado. Primero, una cosmovisión orgánica y dinámica de los problemas sociales y las determinaciones de estos, precisando la magnitud cualitativa y cuantitativa de los mismos, desde los cuales se pretende generar un proceso de intervención profesional. Y segundo, la investigación diagnóstica ofrece una delimitación de los espacios estratégicos para la acción (p. 7).

Aunque el concepto de pobreza ha estado precedido por una notable ambigüedad manifiesta desde su origen, su uso encuentra una justificación en las preocupaciones éticas, por este tema en particular y por la voluntad política de asumir su solución, El concepto de pobreza tiene una connotación claramente descriptiva de una situación social (Altimir, 1979).

La noción de pobreza se basa en última instancia en un criterio de cuáles son los niveles de bienestar adecuados y cuáles las necesidades básicas que deben ser satisfechas. Este criterio es normativo (Altimir, 1979) y alude a las convenciones y estándares sociales en un lugar y tiempo determinados (Sen, 1992). Sin menoscabo de lo anterior existen criterios de referencia de los elementos básicos de bienestar relativos al estilo de vida de las sociedades contemporáneas, que dan cuenta de aquellos asuntos a los cuales todas las personas tenemos derecho y que nacen de la concepción actual de la dignidad y del precedente de universalidad de los derechos humanos (Altimir, 1979).

En América Latina uno de los conceptos más utilizados para el acercamiento a la caracterización de la pobreza es el de insatisfacción de necesidades, esta perspectiva 
consiste en verificar si los hogares han satisfecho una serie de necesidades previamente establecidas y considera pobres a aquellos que no lo hayan logrado.

Para efectos de este estudio consideramos como indicadores de pobreza las carencias que a continuación se señalan y que se han constituido un común denominador de la perspectiva de pobreza descrita desde la insatisfacción de necesidades básicas.

- Hacinamiento.

- Vivienda inadecuada.

- Abastecimiento inadecuado de agua.

- Carencia o inconveniencia de servicios sanitarios para el desecho de excretas.

- Inasistencia a escuelas primarias de los menores en edad escolar.

- No acceso a la salud oportuno y de calidad satisfactoria.

- Condiciones de ingreso y empleo de la población

El acceso a una vivienda adecuada se vincula con la necesidad de las personas de protegerse del medio ambiente, así como con aspectos de privacidad e higiene, cuya ausencia deteriora considerablemente la calidad de vida. El hacinamiento se mide a partir del número de personas por cuarto, mientras que la calidad de la vivienda se determina en función de los materiales de construcción utilizados en piso, paredes y techo (CEPAL / PNUD, 1989).

La disponibilidad de agua y el acceso a servicios sanitarios básicos se refieren a la disponibilidad, al abastecimiento permanente de agua de buena calidad y en cantidad suficiente, para satisfacer las necesidades de alimentación e higiene y se deduce a partir de dos características: la potabilidad del agua y la forma en que esta es suministrada a la vivienda. En el acceso a servicios sanitarios también se distinguen dos características: la disponibilidad de servicio higiénico y el sistema de eliminación de aguas servidas.

La educación básica constituye un requerimiento mínimo para que las personas puedan incorporarse adecuadamente a la vida productiva y social, por lo que se le considera una necesidad básica. En este caso no solo es importante la asistencia a un establecimiento de educación, sino también la calidad de la educación que allí se genera.

La capacidad económica no mide una necesidad básica propiamente, sino que intenta reflejar la probabilidad que tiene el hogar de obtener recursos suficientes para su consumo, hace referencia a los índices de ocupación y el nivel de ingresos de los pobladores del municipio.

Se plantea que la perspectiva asumida para describir la pobreza en el municipio de Guamal, desde la información de insatisfacción de sus necesidades básicas, ofrece una caracterización de la situación en la que viven los hogares con carencias, lo cual 
es muy útil en el diseño e implementación de políticas focalizadas que apunten a aliviar determinadas necesidades básicas (Feres \& Mancero, 2001).

\section{Resultados}

El municipio de Guamal se encuentra ubicado a $396 \mathrm{~km}$ aproximadamente de Santa Marta, ciudad capital del departamento, localizado en la subregión Sur de Magdalena, se compone en su cabecera de 11 barrios y en la zona rural de 22 corregimientos y 29 veredas.

El municipio tiene una extensión aproximada de $554 \mathrm{~km} 2$, representa el 2,3\% de la superficie del departamento de Magdalena. La comunicación con la capital del departamento se realiza a través de la vía Guamal-Astrea-Arjona-Bosconia-Santa Marta. La malla vial local e intermunicipal permanece en deficiente estado, una que otra calle del casco urbano se encuentra pavimentada.

En Guamal, las actividades económicas de mayor importancia son: el comercio en la cabecera, y la agricultura, la ganadería y la pesca en la zona rural. En el campo se cultiva mayormente: maíz, yuca y en menor escala frijol. Se destaca la ganadería vacuna y la producción y comercialización de cítricos (Alcaldía de Guamal, s.f.).

\section{Características demográficas de la población de Guamal}

El municipio de Guamal para el 2014 contaba con 28.940 habitantes, según los datos arrojados por el Sisbén (corte a diciembre de 2014); con un crecimiento promedio de $1.41 \%$ por año respecto a la cifra del DANE del Censo Nacional de 2005, donde se registró una población de 25.508 habitantes.

La población se distribuía de la siguiente forma: el $29.6 \%$ en la cabecera municipal, el $54.3 \%$ en el centro poblado, que corresponde a los corregimientos, y un $15.9 \%$ localizada en zona rural dispersa, que pertenece a las distintas veredas del municipio. En la zona rural se concentraba el $70.2 \%$ de la población, situación muy similar a la cifra arrojada por el DANE en el Censo Nacional 2005.

La distribución de la población por edad en el municipio de Guamal en dicho censo era la siguiente: 16.814 jóvenes (menores de 30 años) que representaba el $58.1 \%$; la población adulta (de los 30 a 59 años) representaba el $28.42 \%$, y la población de la tercera edad (de 60 años y más) representaba el 13.48\%. Se observa que la población de Guamal es predominantemente joven, lo que plantea demandas de inversión significativas en materia de educación y para la población adulta se plantea necesidades importantes en materia de empleo.

En cuanto a la distribución de la población de Guamal por sexo el 48.3\% estaba constituida por mujeres y el $51,7 \%$ por hombres. En lo relativo al estado civil 11.235 
personas eran solteras (49\%), 6.293 estaban en unión libre (27,4\%), 3.248 estaban casados (14,16\%), 1.305 estaban separados o divorciados (5,7\%) y 849 eran viudos $(3,7 \%)$.

Según estadísticas del Sisbén, en el 2014 Guamal contaba con 6.729 hogares, el $66.86 \%$ de los cuales estaban ubicados en zonas rurales y el otro $33.14 \%$ correspondían a la población localizada en la cabecera. La presencia de un número más significativo de población rural, supone la necesidad de descentralizar los bienes y servicios que se ofrecen a esta población.

En relación con el nivel educativo alcanzado por la población, se identificó que el $11,46 \%$ no había alcanzado ningún nivel educativo, un 46,64\% alcanzó la primaria, un $37,9 \%$ la secundaria, solamente el 1,01\% la técnica o tecnológica, un 2,64\% universitaria y un 0,34\% alcanzó algún nivel de posgrado. Estas estadísticas educativas sugieren necesidades de inversión para superar las carencias, en cuanto a niveles superiores de educación y a quienes no tienen ningún nivel educativo.

\section{Escolaridad}

De 1.656 niños que estaban entre los 3 y 5 años de edad, el 18,3\% frecuentaban algún establecimiento educativo. La población infantil de 6 a 10 años constituida por 3.127 niños, de los cuales 830 no frecuentaban ningún establecimiento escolar; 616 asistían a centros de atención u hogares del ICBF; 760 iban a alguna guardería, sala-cuna, preescolar, jardín infantil, del sector público y 921 asistían a la escuela o colegio.

De los 11 a los 17 años, que son 4.653 adolescentes, 92 no frecuentaban ningún establecimiento educativo. De la población de los 18 a 26 años, el cual correspondía a 5.090 personas, se destaca que 2.966 asistían a un colegio técnico universitario o a una universidad pública; y 22 asistían a una institución privada. Resulta preocupante que solo 5 personas de esta población se estuvieran formando en el SENA, y se resalta el hecho de que 2.094 jóvenes no asistieron a ningún establecimiento académico.

Del grupo de población de 27 años y más, que comprendía 10.462 personas, únicamente 301 estaban preparándose a nivel técnico o profesional.

Los datos de escolaridad resaltan la necesidad de desarrollar estrategias formativas, accesibles para la educación superior de los estudiantes de Guamal y la necesidad de fortalecer los mecanismos de inclusión educativa para preescolares, escolares y jóvenes que se encuentran por fuera del sistema educativo.

\section{Condiciones económicas}

En el municipio de Guamal predomina el estrato uno, al cual pertenecían 21.027 habitantes, es decir, el 72,66\%; al estrato cero 5.808, el 20,07\%; y al estrato dos, 
2.001, el 6,91\%. En Guamal, el 73,12\% de las viviendas pertenece al estrato uno, las viviendas con esta clasificación se ubican principalmente en el centro poblado $42,46 \%$ y en la cabecera $27,37 \%$.

En el último mes se destaca que el 18,2\% de las personas del municipio trabajaban, el 35,55\% se encontraba estudiando y el 19,09\% se dedicaba a los oficios del hogar. Es importante destacar el bajo número de personas jubiladas, que son el $0.16 \%$ de lo habitantes, así como también el alto porcentaje de personas que se encontraban sin realizar actividad alguna, el 25.68\%.

En Guamal, de 15.392 personas que se encuentran en edad productiva (18 a 64 años de edad), el 19,16\% de la población declararon no haber realizado ninguna actividad económica o haber buscado trabajo en el último mes; esto puede ser una clara muestra del índice de desocupación del municipio. Las tasas más altas de desocupación se encuentra entre la población joven de los 18 a 34 años de edad, es decir, 1.789 , representando el $61 \%$ total de la desocupación. Igualmente, un $24,3 \%$ la tasa de desocupación se presenta en la población en edad productiva intermedia (35 a 51 años de edad).

El 39,88\% de la población de Guamal percibía algún tipo de ingreso económico proveniente de actividades laborales, arriendos, subsidios, transferencias y pagos en especie; por el contrario, el $60,12 \%$ de los habitantes, no percibían ningún tipo de ingreso.

\section{Acceso a la salud}

La Organización Mundial de la Salud - OMS (2015) establece que la salud es un derecho fundamental de todo ser humano. El derecho a la salud comprende: el acceso a una atención sanitaria oportuna, aceptable, asequible y de calidad satisfactoria. En su explicación la OMS amplía el concepto de lo que es el acceso a la salud como derecho: Significa que los gobiernos deben crear las condiciones que permitan a todas las personas vivir lo más saludablemente posible. Esas condiciones incluyen la disponibilidad garantizada de servicios de salud, condiciones de trabajo saludable y seguro, vivienda adecuada y alimentos nutritivos. Para efectos de este trabajo se ha considerado la vinculación a servicios de salud, bien sea en el régimen contributivo o el subsidiado.

Según los datos estadísticos encontrados en la base de datos del Sisbén, el 74,1\% de la población del municipio estaba afiliado al régimen subsidiado de salud; el $3,81 \%$ de la población hace parte del régimen contributivo, y el $20,87 \%$ señala no estar afiliado a ningún régimen de salud. El centro poblado (corregimientos) recoge la mayor cantidad de personas afiliadas al régimen subsidiado con 11.947, seguido de la cabecera con 6.156 y, por último, el rural disperso (veredas) con 3.334 personas afiliadas. En la cabecera municipal se presenta el mayor número de afiliados al régi- 
men contributivo con 736, seguido del centro poblado con $325 \mathrm{y}$, por último, el rural disperso con 42 personas afiliadas.

De 20.283 personas que corresponden a la población rural, sumando centro poblado y rural disperso, un 22,6\% declara no tener ningún tipo de afiliación a salud. Además, en la cabecera la población que se declara sin ningún tipo de afiliación, es el $16,76 \%$; esto constituye un motivo de preocupación por el alto porcentaje de población en condición de desprotección.

\section{Perfil habitacional y de saneamiento básico del municipio de Guamal}

En un territorio las relaciones cambiantes y en constante movimiento pueden ordenarse para su representación y análisis en una matriz de doble entrada, en la cual se expresan las tensiones generadas por la población sobre el medio, así como las tensiones generadas por el medio sobre la población, para construir su perfil habitacional y de saneamiento básico (Ministerio de Ambiente, Vivienda y Desarrollo Territorial, 2004).

Utilizando una matriz para el procesamiento del conjunto de información con la que se cuenta sobre los corregimientos y la cabecera municipal de Guamal en cuanto a: población, número de habitantes, número de viviendas, número de hogares, tenencia de la vivienda y los datos respecto a acueducto, aguas residuales y disposición de desechos sólidos, se han podido establecer unos índices de hacinamiento y saneamiento básicos.

Para efectos de representar gráficamente los resultados del análisis de la matriz enunciada se utilizó una herramienta que se ha denominado "semáforo" y que se constituye en un instrumento que da cuenta en la cabecera municipal y en los corregimientos de las situaciones de hacinamiento, saneamiento básico y vivienda, mostrando con color rojo las situaciones críticas que requieren una prioritaria intervención, en amarillo las situaciones intermedias que generan alerta y en verde las situaciones que permiten un plazo más amplio en la intervención, denominadas aceptables.

\section{Perfil habitacional y de saneamiento básico rural}

\section{- Convenciones de la matriz del semáforo rural}

Saneamiento básico: No tiene un servicio o no goza de cobertura, Rojo más del $50 \%$; amarillo entre el $25 \%$ y $50 \%$ y verde cuando es menos del $25 \%$.

Tenencia de la vivienda: No tienen vivienda propia, Rojo cuando es más del $40 \%$; amarillo entre $25 \%$ y $40 \%$ y verde menos del $25 \%$. 


\section{- Perfil habitacional rural}

De acuerdo con lo encontrado en el procesamiento de la información a través de la matriz, para el análisis de aspectos socioeconómicos en los 22 corregimientos del área rural de Guamal habitan 20.290 personas, un promedio de 1.13 núcleos familiares (hogares) por vivienda.

El promedio de personas que habitan por vivienda corresponde a 5.12. Los corregimientos donde es mayor este promedio son: Playas Blancas con 6.28; Villanueva 5.86; y Sitio Nuevo con 5.74 personas por vivienda. De un total de 4.481 núcleos de vivienda (hogares) de la zona rural, 521 viven en hacinamiento, o sea alrededor de un $12 \%$. En los corregimientos donde más se presenta este fenómeno son: Playas Blancas con el 25\%, San Antonio con 23\% y Santa Teresa con el 22\%.

El déficit de vivienda en la zona rural de Guamal es del 38\%, entre los corregimientos donde hay más carencia están Santa Teresa con el 54\%, Ricaurte con 48\%, San Isidro y Bellavista con el $45 \%$. Entre los corregimientos donde el déficit de vivienda es menor están: Las Flores con el 28\%; Guaimaral con el 30\% y La Ceiba con el 31\%. El déficit de vivienda y hacinamiento en la zona rural de Guamal es preocupante desde la perspectiva de sus consecuencias para la posibilidad de acceder a una vida digna, con calidad y mejores condiciones de convivencia intrafamiliar.

\section{- Saneamiento básico rural}

Para Colombia en el 2008 la cobertura global de agua potable se había estimado en $91,5 \%$ y la de alcantarillado en $87,4 \%$. En la zona urbana se estimó en 97,6\% para acueducto y en $92,9 \%$ para alcantarillado, mientras que en la zona rural es de $72,0 \%$ y $69,6 \%$, respectivamente, reflejando la gran disparidad que existe en el país entre las zonas urbana y rural (Gobierno de Colombia, 2011). No obstante lo anterior en Guamal los datos a este respecto son los siguientes.

Suministro de agua: en la zona rural del municipio a nivel de saneamiento básico se evidencia una situación crítica. El semáforo se ubica entre amarillo y rojo en la generalidad de los corregimientos. En el 2014 en el área rural el 68\% de las personas no contaban con suficiente cantidad y calidad de agua. Así mismo, las personas en sus viviendas no contaban con acceso a ningún tipo de acueducto por muy rudimentario que sea, por tanto, las personas obtienen de otras maneras el suministro de agua no tratada (pozo, jagüey, aljibe río o ciénaga); con los riesgos evidentes que ellos implican para la salud. Los únicos corregimientos que se ubican en situación aceptable son: Guaimaral, Ricaurte, Pam Pam, Hatoviejo y Murillo. Como puede observarse la disponibilidad de acueducto en Guamal es inferior al promedio señalado para el área rural a nivel nacional.

Aguas residuales: en el servicio de alcantarillado la situación en la zona rural también se muestra crítica. El 51,5\% de las personas tiene una inadecuada eliminación de excretas (hace referencia que el $51,5 \%$ de la población no tiene servicio 
sanitario, tiene letrina con uso inadecuado, o inodoro sin conexión a alcantarillado o pozo séptico). Los únicos corregimientos que presentaron condiciones aceptables fueron Ricaurte, Murillo y Bellavista.

Desechos sólidos: el indicador del número de viviendas sin servicio de recolección de basuras mostró que para el 2014 el 100\% de la población de la zona rural del municipio no contaba con el servicio de recolección de basura y, lo que es peor, la población en su totalidad deposita la basura bien sea a campo abierto (fuentes hídricas, patios, zanjas, etc.) o la quema, resultando estas formas de disposición perjudiciales para el medio ambiente y para la salud.

\section{Perfil habitacional y de saneamiento básico urbano}

\section{- Convenciones de la matriz del semáforo urbano}

Hacinamiento, características y tenencia de la vivienda: hace referencia a la presencia de un porcentaje determinado de viviendas en cada una de las variables analizadas. Rojo, más del 30\%; amarillo, entre el 10\% y el 30\% y verde, menos del $10 \%$.

Saneamiento básico: para el indicador de saneamiento el semáforo cambia el criterio: cuando un porcentaje $\%$ de las viviendas no poseen el servicio. Rojo, más del 15\%; amarillo, entre el 5 y el 15\% y verde, menos del $5 \%$.

\section{- Perfil habitacional urbano}

Hacinamiento: la cabecera del municipio de Guamal está conformada por 11 barrios, donde habitan 8.557 personas. La población urbana del municipio comprende 2.230 núcleos familiares que se distribuyen en 2.026 viviendas (1.10 núcleos familiares por vivienda).

En el nivel de hacinamiento en las viviendas de la cabecera se presenta una situación aceptable, con el 9\%. Tres barrios se ubican en situación de alerta (entre el 10 y el 30\% de las viviendas) y son: el barrio Diez de Marzo, con el 13\%; Lara, con 12\%; y San Martín, también con el 12\%.

En lo que se refiere al promedio de personas que habitan la vivienda corresponde a 4,2. Los barrios donde este indicador es mayor son: Brisas de Río con 4,7; San Martín con 5,07 y Urbanización el Porvenir con 5,36.

Déficit de vivienda: el déficit de vivienda en la zona urbana es del $42 \%$, lo que quiere decir que 945 familias carecen de vivienda propia. Tal situación es considerada crítica, encontrándose los peores registros en la Urbanización El Carmen con el $71 \%$; barrio Abajo con 47\% y en barrio Centro donde llega al 43\%. El único barrio en el que el déficit de vivienda no aparece en situación crítica es la Urbanización El Porvenir, que se encuentra en situación de alerta con el $28 \%$. 
Características de las viviendas: con relación al indicador de materiales adecuados de las viviendas, en lo que tiene que ver con paredes, pisos y techos, en general el semáforo muestra situaciones aceptables en las viviendas, en particular en cuanto a paredes y techo. Sin embargo, las condiciones son críticas en lo relacionado con la carencia de material adecuado en los pisos, el 21\% se ubica entre situación de alerta y crítica. Los barrios con mayor déficit en este aspecto son: Brisas del Río con el 77\%; Urbanización El Porvenir con el 67\%; San Luis con 58\%; Doce de Octubre con $56 \%$ y San Martín con el 43\%. Los barrios que están en situación de alerta son Diez de Marzo y Urbanización Simón Bolívar, ambos con el 17\%, y Lara con el 16\%. Dicha condición muestra la gravedad del problema habitacional de carácter cualitativo en la zona urbana del municipio.

Sumado a lo anterior, es importante resaltar lo relativo a la tipología de la vivienda. En la zona urbana 769 viviendas son tipo cuarto, lo que constituye un 38\%, siendo esta condición mayor en el barrio Brisas del Río con el 60\% y la Urbanización Simón Bolívar con el 50\%. Esto también es indicador del déficit habitacional en el municipio.

Saneamiento básico urbano: las condiciones de saneamiento básico en la cabecera del municipio de Guamal son aceptables, presentándose barrios donde la situación es deficiente. Frente al servicio de acueducto, en la zona urbana el 3\% de las viviendas no cuentan con este servicio. Entre los barrios de mayor vulnerabilidad están: el barrio El Porvenir con un 10\% y Brisas del Río, donde el nivel de carencia llega al 30\%.

En la cabecera el panorama es lamentable en lo referente al acceso al servicio de alcantarillado. El 6\% de las viviendas no cuentan con este servicio. Se evidencia como crítico en los barrios Brisas del Río y Urbanización El Porvenir, donde la carencia alcanza el $47 \%$ y el $31 \%$, respectivamente.

Con relación al servicio de recolección de basuras en la cabecera, la mayoría de las viviendas están entre situación de alerta y crítica, las zonas más problemáticas son los barrios Brisas del Río con el 26\%, Doce de Octubre con el 19\% y la Urbanización el Porvenir con el 15\%.

Aunque las condiciones de saneamiento básico en el área urbana son mejores que en el área rural, es necesario destacar que en la zona urbana es grave el déficit de vivienda, la calidad de los materiales de construcción en pisos y la carencia de servicios de disposición de aguas residuales y desechos sólidos.

\section{- Convivencia participación y seguridad}

En cuanto a la participación de la población en la cabecera municipal, se pudo establecer que las actividades deportivas como el fútbol y las actividades religiosas son elementos que la facilitan, también en el municipio se han conformado juntas de acción comunal y sindicatos; conocen algunos mecanismos de participación ciudadana pero aceptan que poco los han utilizado. 
En la zona rural los habitantes participan en fiestas patronales, actividades deportivas como el fútbol y baloncesto y actividades culturales, también han conformado juntas de trabajo comunitario. Conocen algunos medios de participación ciudadana. Algunos habitantes han realizado movilizaciones para obtener respuesta a sus necesidades. Mientras otros habitantes manifiestan no haber participado en ningún tipo de movilización social.

Con relación a la seguridad (percepción de ausencia de riesgo o la confianza en algo o en alguien) las personas de la cabecera hoy se sienten más inseguras de salir en las noches, sintiéndose más vulnerables las mujeres. Al igual que en la cabecera municipal en los corregimientos en general, los habitantes manifestaron que se sienten inseguros, pues temen trasladarse a la cabecera municipal por las noches; aunque la percepción de inseguridad no es unánime.

Estas apreciaciones acerca de la seguridad fueron complementadas con algunas informaciones que aportaron la entrevista con la inspectora de Policía de la cabecera municipal y la consulta a los datos estadísticos del Ministerio de Defensa Nacional, planteados en el informe del comportamiento delincuencial, resultado de denuncias ante autoridad competente desde el 2014 hasta julio de 2015. La información obtenida respecto al comportamiento delincuencial en el municipio, fue la siguiente: en el 2014 se presentaron 4 homicidios y 3 en el 2015, en estos años se denunciaron 6 hurtos (personas, residencias, comercio), se presentó el hurto de una motocicleta.

Según la percepción de la inspectora de Policía la seguridad en el municipio, se ve afectada por unos factores recurrentes:

- Las lesiones comunes resultado de riñas callejeras, relacionadas con el consumo de alcohol.

- El contrabando de gasolina (2.700 galones decomisados en el 2014) y otros bienes tales como pólvora, armas blancas, motocicletas.

- Los accidentes de tránsito que ocasionan muertes y lesiones y están originados en el exceso de velocidad, el consumo de licor mientras se conduce, el mal estado de vehículos y motos y de las vías, considerándose como crítica la vía Guamal-Astrea.

- Las incursiones periódicas de paramilitares en la zona que generan extorsiones y vacunas (Martínez, Comunicación personal, 7 de julio de 2015).

En cuanto al asunto relativo, la confianza (entendida como esperanza firme que una persona tiene en que otra persona o institución funcione, o actúe en términos de mejorar la calidad y condiciones de vida y de convivencia en la comunidad) tanto en la cabecera municipal como en los corregimientos, los participantes manifestaron tener poca confianza en la alcaldía, la policía y los partidos políticos. Existe mayor confianza en la iglesia y en la escuela. 


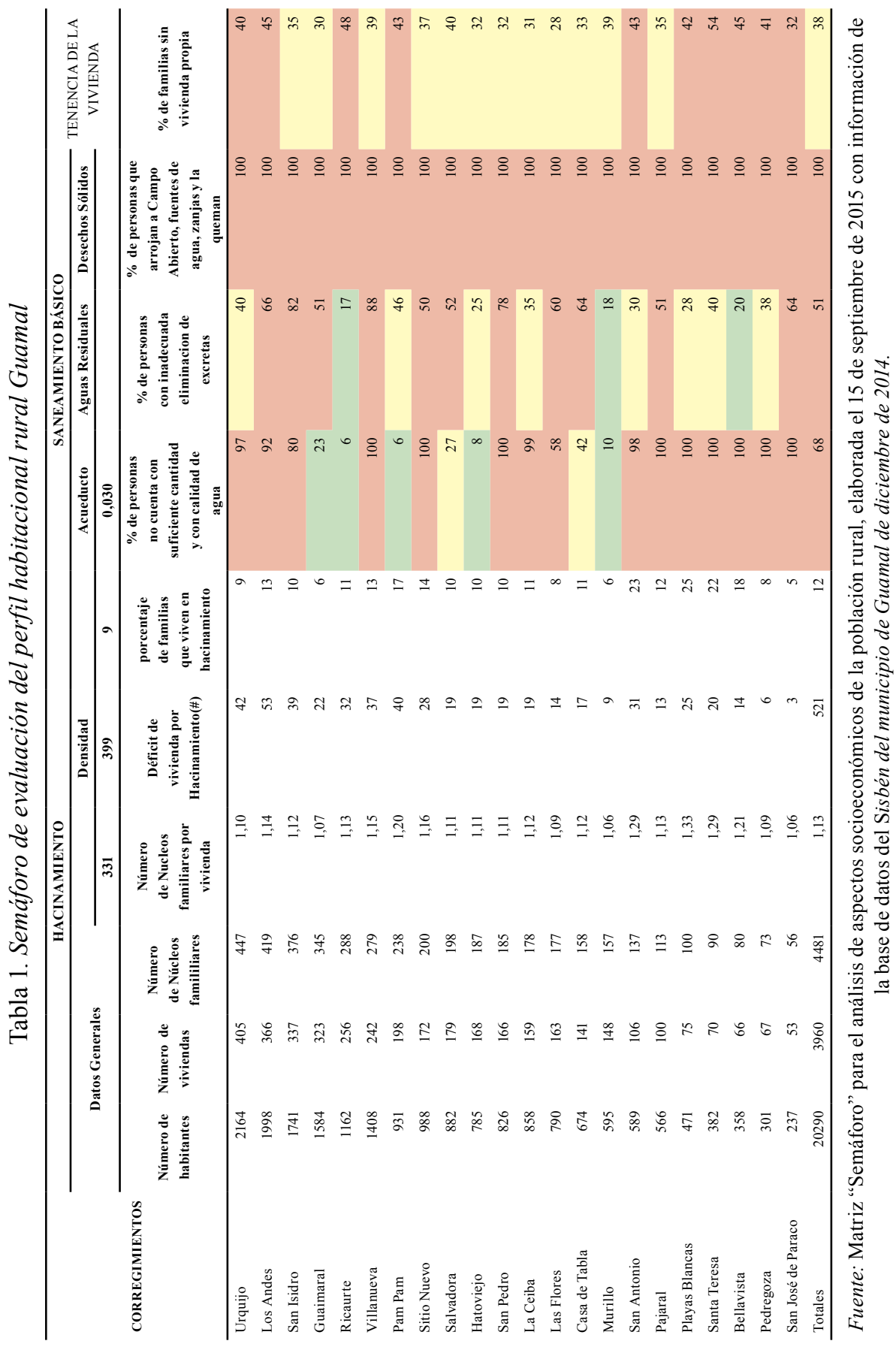


Diagnóstico social, demográfico y económico, y situación de pobreza...

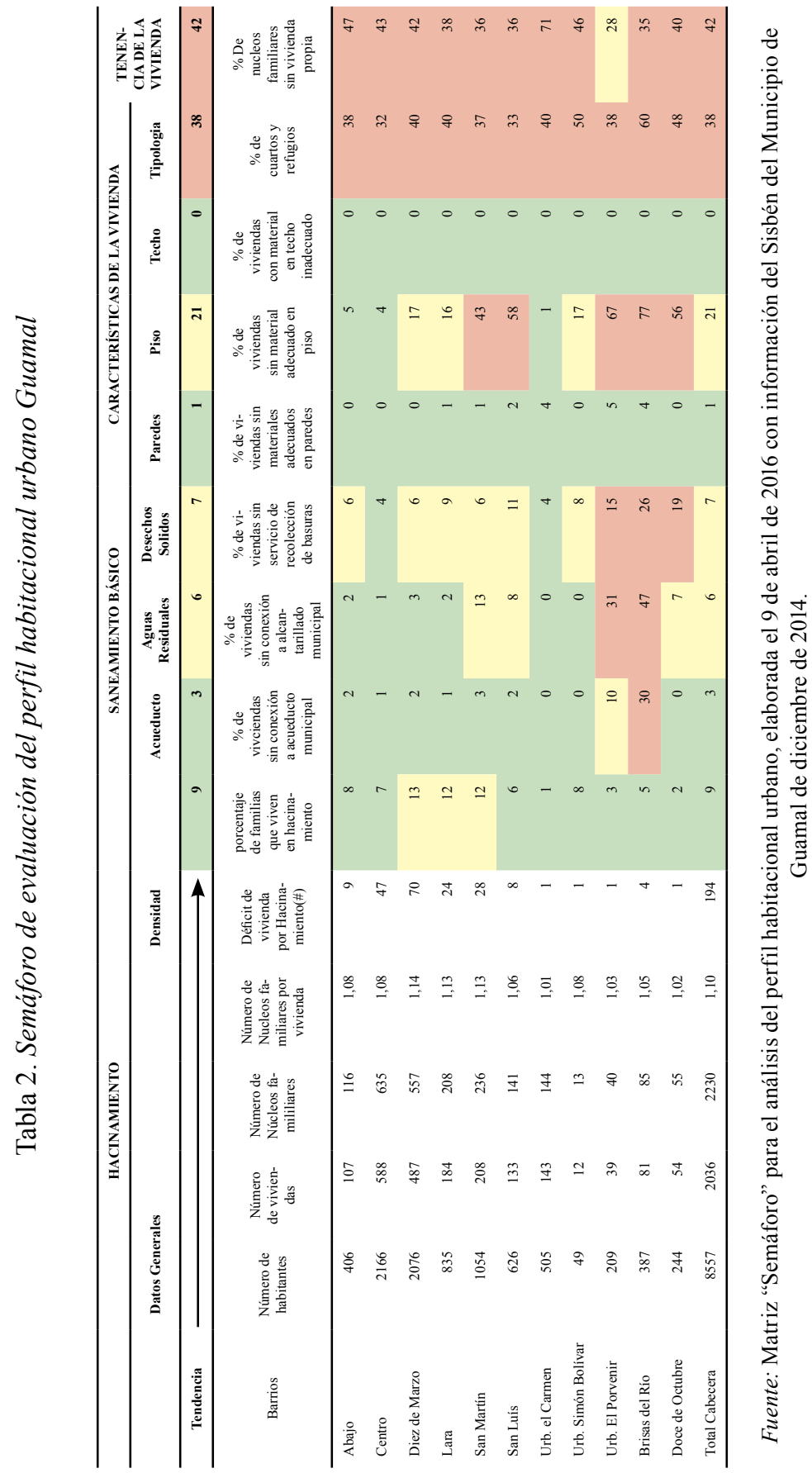




\section{Conclusiones}

El municipio de Guamal ha tenido desde el 2005 un crecimiento poblacional promedio de $1.41 \%$ por año, mayor que el del país, del que se reporta un índice de crecimiento de 1.16 (Index Mundi, 2017). La población en edad dependiente de Guamal constituye el $40.5 \%$ del total de habitantes, siendo un $30 \%$ menores de 15 años y un 10.5 mayores de 60 años, lo que nos plantea una relación de dependencia de 43.44 por cada 100 habitantes $^{6}$ (Ministerio de Salud y Proteccion Social, 2013).

La población se encuentra ubicada en un $70.4 \%$ en el área rural del municipio. Según estadísticas del Sisbén, el promedio de personas que viven por hogar en el municipio es de 4,3\%, promedio mayor al del país. El 59.5\% de los habitantes se encuentran en edad productiva (Ministerio de Salud y Proteccion Social, 2013) ${ }^{7}$, pero solo un $40 \%$ percibe ingresos, por esta razón muchos jóvenes y adultos decidan migrar a diferentes ciudades del país, como Barranquilla, Santa Marta, Valledupar, Bogotá, entre otras, para tener la posibilidad de acceder a un trabajo.

La clasificación por estrato del municipio nos lleva a inferir las condiciones de precariedad y vulnerabilidad en las que se encuentra la población, donde el $20,07 \%$ se ubica en estrato 0 y el 72,66\% en estrato 1 . El 74,1\% de la población se encuentra afiliada al régimen subsidiado de salud. Un 20,87\% señala no estar afiliado a ningún régimen, esto es preocupante por el alto porcentaje de población en condición de desprotección.

Los habitantes consideran que las personas no están afiliadas a salud por desconocimiento del proceso y del servicio, quienes tienen el servicio lo consideran deficitario, pues se presentan problemas en la oportunidad de la atención, calidad de los medicamentos y acceso al servicio por lo apartado de su ubicación.

De la población de Guamal de 10 años o más, según el Sisbén, un 11,46\% no ha alcanzado ningún nivel educativo, aunque se ha logrado mayor cobertura en la educación primaria y secundaria sin lograr el $100 \%$, además, es necesario ampliar las posibilidades de formación en educación superior, que le posibiliten a la región y a la población, mayor desarrollo y mejores condiciones de vida. Considerando el análisis elaborado por Amartya Sen respecto a la relación educación-pobreza, la primera busca la expansión de las capacidades humanas, con el fin de que los individuos tengan la libertad para escoger una manera de vivir valiosa desde su perspectiva, en este planteamiento, el fin es el desarrollo del ser humano. Por esto la educación es una herramienta muy importante para la reducción de la pobreza, ya que contribuye a acrecentar el capital humano y tiene efectos sobre las capacidades y habilidades

6 La relación de dependencia total se establece por la suma de la población menor de 15 años más la población de 60 años o más (demográficamente no activos, aunque en la realidad socioeconómica sí lo sean), sobre la población en edad de trabajar (o población activa), es decir, sobre el total de la población ente los 15 y 59 años de edad. Muestra una aproximación a la carga que ejercen las personas potencialmente dependientes - niños, adolescentes y personas mayores- sobre las personas que son potencialmente activas y pueden aportar los recursos para atender sus demandas.

$7 \quad$ Población entre 15 y 60 años. 
de las personas; las capacidades y habilidades de las personas tienen una relación directa con su libertad y bienestar y además influyen en la productividad económica y en el cambio social (Sen, 1998).

Desde otro ángulo se señalan algunas relaciones conflictivas entre la educación y la pobreza, señalando cómo, si bien la educación no logra que las personas salgan de la pobreza, tampoco esta puede dejarse a un lado cuando se quiere reducir la pobreza y se plantea además, que al caracterizar a los pobres estos son "quienes no acceden a la escuela, aceden en circunstancias inequitativas o reciben una educación de baja calidad" (Bradesch, 2011, p. 77).

En Guamal existen serias carencias en lo relativo a acueducto, alcantarillado y recolección de basuras, situaciones estas que perjudican las condiciones de salud pública en la zona. Comparando la zona urbana con la rural, la segunda se ve mucho más afectada en sus condiciones de vivienda y saneamiento básico.

El derecho al techo ha sido relacionado con el derecho a la salud y desde esta perspectiva, la vivienda tiene que cumplir con unas condiciones básicas de salubridad. Cuando se alude específicamente al derecho a la vivienda se plantean criterios que superan las características particulares de construcción (materiales, ventilación, luminosidad) y se incluyen aspectos como su entorno en cuanto a seguridad, infraestructura, servicios, accesibilidad, entre otros, en síntesis se señala al hablar del tema la importancia de un hábitat y una vivienda digna.

En otras palabras, la expectativa frente a un nivel de vida digno sería que: la vivienda se constituya en un "lugar con privacidad, espacio suficiente, accesibilidad física, seguridad adecuada, seguridad de tenencia, estabilidad y durabilidad estructural, iluminación, calefacción y ventilación dignos; una infraestructura básica que incluya abastecimiento de agua, saneamiento y eliminación de desechos; factores apropiados de calidad del medioambiente y de salud y un emplazamiento adecuado y con acceso a fuentes de trabajo y a los servicios básicos, todo ello a un costo razonable" (Giraldo, Torres \& Bateman, 2004, p. 19).

Entre todos los factores que constituyen una vivienda deficitaria, el hacinamiento tiene particular importancia, pues este elemento incide en la privacidad y la libre circulación (Chapin, 1963 citado en Lentini \& Palero, 1997) los cuales contribuyen a preservar la salud mental, pues la privacidad es necesaria para que los subsistemas familiares (parental, paterno-filial, filial) puedan sostener relaciones adecuadas, con cercanía, pero con independencia y para la realización de manera sosegada y autónoma de las actividades cotidianas, comer, dormir, preparar los alimentos, recrearse, entre otras (Lentini \& Palero, 1997).

La libre circulación en la vivienda evita el estrés sicológico, que supone estar constreñido en un espacio. Existen investigaciones realizadas que permiten inferir estas situaciones, como puede observarse en estudios anteriores, como el que aparece en el artículo "Algunas características del maltrato físico al niño en nuestro medio", 
sugieren que en el grupo de niños estudiados, el maltrato ocurre en familias con condiciones de vivienda deficitaria (Alfonso, Fernández, Perea \& García, 2004) y en el artículo "Habitabilidad de la vivienda ,estrés y violencia familiar" donde se encontró que algunos aspectos de la deficiente habitabilidad de la vivienda produce estrés en sus moradores y éste influye en la violencia intrafamiliar (Corral, Barron, Cuen \& Tapia, 2011).

La carencia de agua potable en Guamal es uno de los asuntos más preocupantes, dado que el acceso a esta es un derecho y que si consideramos la pobreza como una imposibilidad de ejercer capacidades y libertades sustanciales, el no poder acceder al agua potable es la privación del ejercicio de una oportunidad necesaria para la vida, pudiendo afirmarse que la pobreza entraña - en buena medida- la carencia de este elemento, y esta situación a su vez supone pobreza (Correa, 2017).

En Colombia el costo social relacionado con el agua se manifiesta impactando las condiciones de salud, mortalidad y morbilidad, cuyo origen es el consumo de agua contaminada, "generando pérdidas del 2.8\% de PIB (Producto Interno Bruto)", (Sánchez, Ahmed, \& Awe, 2006, p. 118) lo que acentúa la pobreza y afecta el desarrollo nacional (Sánchez, Ahmed, \& Awe, 2006).

Las deficiencias en el acceso al agua, afectan aspectos como la salud y en consecuencia otros asuntos como el empleo y los ingresos familiares, presentando un impacto en el bienestar general de las poblaciones (Angulo, Díaz, \& Pardo, 2011). "El acceso al agua es un eje fundamental en el desarrollo que contribuye a superar condiciones como la desigualdad y la vulnerabilidad socioambiental" (Correa, 2017, p. 40).

La población participa de actividades deportivas, en actividades religiosas y se ha integrado a juntas de acción comunal y comités de salud. Reconocen los mecanismos de participación pero aceptan que poco los han utilizado. Han realizado pocos movimientos de participación popular esporádicos, principalmente, en torno al reconocimiento de derechos en salud, educación y servicios públicos. Según estudios realizados la población pobre tiene menores índices de participación, expresada no solo en sus niveles de votación sino también en su vinculación con procesos colectivos más informales y menos jerárquicos, más esporádicos que sistemáticos lo que también plantearía una cercanía de la población con la condición de pobreza.

En cuanto al asunto relativo a la confianza en autoridades e instituciones en la cabecera municipal y en los corregimientos, se tiene poca confianza en la Alcaldía, la Policía y los partidos políticos, pero existe mayor confianza en la iglesia y en la escuela. En diversos textos científicos de literatura del desarrollo ha ido tomando fuerza la idea de que la pobreza también tiene consecuencias psicológicas y sociales (Ernst \& Haushofer, 2014), una de las cuales sería la pérdida social de confianza (Zak \& Nack, 2001). La confianza se ha considerado fundamental, por facilitar la integración social, promueve el crecimiento económico (Algan \& Cahuc, 2010) (Bjørnskov, 
2012), incrementa la participación cívica (La Porta, Lopez de Silanes, Sheleifer \& Vishhny, 1997) y reduce el crimen (Kawachi, Kennedy, Lochner \& Prothrow, 2011).

La situación de las familias de Guamal se caracteriza también por la tendencia al incumplimiento de las responsabilidades alimentarias por parte de los padres, situaciones de abuso sexual a menores por parte de miembros de la familia (padres, padrastros, tíos...). La información a este respecto es pobre y fragmentada, lo que permite inferir una situación de abandono del Estado frente a la población en lo relativo a estos asuntos, a pesar de que existe una comisaría de familia en el municipio (Rivera, Comunicación personal, 7 de julio de 2015).

El municipio de Guamal, según lo observado en su diagnóstico, presenta una serie de carencias relativas a sus condiciones de vivienda, saneamiento básico, empleo, educación, salud, participación y confianza en sus instituciones. Características todas estas asociadas a la concepción de pobreza como carencia en la satisfacción de necesidades que hemos venido explorando en este texto, por lo tanto podríamos afirmar que esta población es pobre, pero no se puede desconocer que la condición de pobreza del municipio da cuenta de una situación más general que es la pobreza en el sector rural del país, contrastando la situación de Guamal con las características generales del sector rural se podría señalar que Colombia al ser uno de los países de América Latina que actualmente cuenta con un número considerable de población rural, al igual que otros países como Bolivia, Perú, Nicaragua, Costa Rica, Honduras, afronta la situación de un significativo grupo de población que vive en condiciones de extrema pobreza y miseria (Pérez \& Pérez, 2002), en la que lo verdaderamente preocupante no es que la pobreza rural sea mayor que la pobreza urbana sino que cada vez la brecha se amplíe, porque se evidencia un empobrecimiento mayor y más rápido en las áreas rurales que en las urbanas (Perry, 2010) (Departamento Nacional de Planeación, 2015).

Cerca de las dos terceras partes de los moradores rurales en Colombia son pobres y más de la tercera parte de los pobres rurales se hayan en la indigencia (Perry, 2010, p. 9).

En Colombia como en el resto de Latinoamérica la pobreza tiene un marcado carácter rural (Perfetti, 2009).

La pobreza rural en el país se manifiesta principalmente en la dificultad para acceder a los servicios de salud, educación, seguridad social y servicios públicos (Pérez \& Pérez, 2002), "siendo la cobertura de servicios sociales mucho menor en el sector rural que en el urbano, de forma tal que de 1.066 .157 entre niños 5 y 16 años que están por fuera del sistema escolar el $70 \%$ se encuentra en el campo" (Perry, 2010, p. 9).

“En la educación superior la situación es dramática, ya que solo el 0,76\% de la población ha alcanzado estudios universitarios, en comparación con el sector urbano donde esta cifra es 9,49\%. Dicho en otros términos, por cada persona que ha 
alcanzado un nivel universitario en el campo hay 31 personas en la ciudad con título profesional" (Pérez \& Pérez, 2002, p. 48).

La falta de equidad entre lo urbano y lo rural se ve reforzada con el hecho de que un gran porcentaje de docentes del sector rural no tiene educación superior (su educación media para 1995 era de cerca de 12 años) y está por debajo de la educación media de los docentes urbanos (IICA Departamento Nacional de Planeación, 1998) citado en (Pérez \& Pérez, 2002, p. 49). Este hecho contribuye a la baja calidad de la educación rural agravada por la carencia de infraestructura y escasez de materiales, ayudas didácticas y bibliotecas.

Si persisten todas estas inequidades, los habitantes del sector rural nunca podrán competir en el mercado laboral con igualdad de oportunidades respecto a las que tienen los habitantes de las zonas urbanas.

En el sector rural las personas ocupadas no tiene afiliación a la seguridad social, en el 2003 solo el $15.3 \%$ de los hombres y el $20.4 \%$ estaban afiliados a riesgos y el 14.7\% y el 24.4, respectivamente, estaban filiados a pensiones (Perry, 2010, p. 9).

La cobertura de los servicios públicos es baja en el ámbito rural, tienen agua potable solo el 59.8\% de los hogares, alcantarillado el 17.9\% (Perry, 2010, p. 9).

En síntesis la pobreza rural en Colombia es un problema de considerable magnitud, del cual el caso Guamal es un claro representante, dado que allí como en buena parte del país rural se evidencian diagnósticos caracterizados por significativas carencias en la satisfacción de las necesidades y un precario acceso a servicios sociales y públicos, situación que no puede soslayarse en un Estado social de derecho como el colombiano, que en el preámbulo de su Constitución Política señala entre sus finalidades: "fortalecer la unidad de la nación y asegurar a sus integrantes la vida, la convivencia, el trabajo, la justicia, la igualdad, el conocimiento, la libertad y la paz, dentro de un marco jurídico, democrático y participativo que garantice un orden político, económico y social justo" y plantea entre sus principios fundamentales: "Construir una sociedad democrática, participativa y pluralista fundada en el respeto de la dignidad humana" (Constitución Política de Colombia, 1991). Es por lo tanto un deber de la sociedad colombiana en su conjunto superar todo tipo de exclusión, como una cuestión de justicia. Esto como reconocimiento de que todas las personas son dignas y deben ser tratadas con consideración y respeto, para hacer de este país un lugar más humano y más habitable (Martínez, 2002).

Analizando el diagnóstico social del municipio es necesario plantear las siguientes situaciones que son prioritarias para atender:

El déficit del número de viviendas de Guamal que se traduce en hacinamiento, debe ser atendido mediante procesos de cooperación entre el sector público y el privado, que faciliten el desarrollo de procesos de construcción de viviendas de interés social. 
De otro lado, alternativas como la planteada anteriormente mejorarían las condiciones de empleo de la población, que dado su bajo nivel de cualificación podría participar de trabajos en el ámbito de construcción de vivienda y de infraestructura que reduzca las carencias en materia de saneamiento básico y los déficit cuantitativo y cualitativo de las unidades habitacionales existentes, especialmente en términos del material de los pisos.

Es necesario promover convenios entre las autoridades municipales y los centros de educación superior del país que ofrecen programas a distancia en diferentes áreas del conocimiento. Además fortalecer el papel del Sena como ente capacitador en el municipio.

En materia de salud se requiere fortalecer el proceso de afiliación de la población para incrementar el número de pobladores vinculados a los diferentes regímenes de salud y fortalecer los mecanismos de participación de la población en las entidades de salud, a fin de que se haga posible una atención más eficiente y oportuna. Además, proponer estrategias como la de teleasistencia de bajo costo para atención primaria.

Es necesario aumentar la cobertura y la calidad de los servicios de aseo, mediante la promoción de la cultura de la gestión segura de los residuos, logrando que la comunidad asuma una actitud de responsabilidad en su gestión, lo que supondrá sensibilización, educación, formación y apoyo a la población en este aspecto.

Según Foster y Yepes (2006) las dificultades en el acceso a agua potable están relacionadas con la pobreza, la desigualdad y las inequitativas relaciones de poder, por lo tanto en las regiones y países de América Latina y especialmente en Colombia (Foster \& Yepes, 2006), se hace necesaria la participación de la sociedad civil, que permita una gobernanza del agua, para la gestión integral de esta y que impliquen por ejemplo el desarrollo de tecnologías innovadoras para el suministro de agua potable en la zona, como es el caso de extraer la humedad del aire, utilizando energía eólica y potabilizar el agua mediante filtros. Esto se desarrollaría a través de convenios que se establezcan entre el sector público y el privado, y buscando asistencia con organismos extranjeros que han desarrollado proyectos de apoyo a comunidades en este sentido.

A partir de un proyecto educativo, formar en la comunidad personas con pensamiento emprendedor, creativas, recursivas e innovadoras para que logren sacar provecho a los recursos naturales con los que cuenta este municipio desde un enfoque socialmente responsable, y de esta manera lograr formar microempresas con el fin de generar empleo y distribuir sus productos a otras regiones del país.

Motivar y capacitar a las personas para que hagan huertas caseras en sus casas, puesto que la mayoría de las viviendas cuentan con espacio suficiente para realizar este proyecto. 
Los niveles de confianza de la población, al ser mayores frente a instituciones como la Iglesia y la escuela, plantean la necesidad de que estas dos organizaciones emprendan procesos educativos y de movilización de la población en búsqueda de mejores condiciones de vida, así como también que enfaticen su labor educativa y concientizadora en lo relativo a la importancia de la participación y el logro efectivo de reconocimiento de derechos sociales y económicos y el cumplimiento de deberes de convivencia social.

Fomentar el compromiso de las entidades educativas con procesos de conservación del recurso hídrico, educando en torno a fomentar el ahorro y uso racional del agua, en las cuencas de las quebradas, propiciando actividades de recolección de plántulas y semillas de especies nativas y la siembra de árboles.

Frente al mejoramiento de la seguridad en la zona se podría implementar la utilización de APP (aplicación de seguridad) que sirva como alarma comunitaria dentro de una red social privada de vecinos ante una emergencia; en la cual los integrantes pueden enviar alertas a la red, disminuyendo el tiempo de respuesta ante situaciones de emergencia. Además estas APP sirven de medio de comunicación entre las redes vecinales, que permite estar al tanto de noticias de la zona; crear grupos de vecinos intercomunicados (chats) de distintos temas afines a la comunidad; vender o regalar objetos; promover iniciativas vecinales, y fortalecer la convivencia y organización de los vecindarios a través de un medio digital gratuito y democrático.

\section{Referencias}

Alfonso, O., Fernández, G., Perea, J., \& García, F. (2004). Algunas características del maltrato físico al niño en nuestro medio. Revista Habanera de Ciencias Médicas, 3(9). [en línea]. Recuperado de http://www.revhabanera.sld.cu/index.php/rhab/ article/view/696

Algan, Y., \& Cahuc, P. (2010, december). Inherited Trust and Growth. American Economic Review, 100(5), 2060-2092.

Altimir, O. (1979). La dimensión de la pobreza en América Latina. Santiago de Chile: Cepal - Ilpes.

Angulo, R., Díaz, Y., \& Pardo, R. (2011, agosto 26). Índice de pobreza multidimensional para Colombia - (IPM-Colombia) 1997-2010. Recuperado de Archivos de Economía: https://colaboracion.dnp.gov.co/CDT/Estudios\%20Econmicos/382. pdf

Baena, G. (1988). Manual para elaborar trabajos de investigación documental. México: Unidos Mexicanos.

Bjørnskov, C. (2012, abril). How Does Social Trust Affect Economic Growth? Southem Economic Journal, 78, 1346-1368. 
Bradesch, M. (2011). Educación y pobreza: Una relación conflictiva. En A. Ziccardi. Pobreza, desigualdad social y ciudadanía. Los limites de las políticas sociales en América Latina. (pp. 65-78). Buenos Sires: Clacso.

Constitución Política de Colombia. (1991). Bogotá: Corte Constitucional.

Corantioquia. (2003). Proyecto: Propuesta para el manejo de las variables poblacionales en los procesos de desarrollo y ordenamiento territorial: Poblamiento y dinámicas territoriales en la jurisdicción de Corantioquia. Convenio interadministrativo. Conrtrato No. 4300. Medellín: Escuela de Planeación Urbano Regional, Facultad de Arquitectura, Universidad Nacional de Colombia.

Corral, V., Barron, M., Cuen, A., \& Tapia, C. (2011). Habitabilidad de la vivienda, estrés y violencia familiar. Revista Bilingue de Psicologia Ambiental, 2.

Correa, G. (2017, enero-abril). Acceso al agua pobreza y desarrollo en Colombia. Revista de la Universidad de la Salle, 72(72), 27-46.

Chapin, S. (1963). Algunos problemas de la vivienda en relación con la higiene. En R. Merton. Sociología de la vivienda. Buenos Aires: Hombre y Sociedad.

DANE. (2013). Pobreza monetaria y multidimensional en Colombia 2012. Boletín de prensa. Bogotá. Recuperado de https:/www.dane.gov.co/files/investigaciones/ condiciones_vida/pobreza/boletin_pobreza_2012xx.pdf

DANE. (2014). Magdalena: Pobreza monetaria 2013. Boletín de prensa. Bogotá. Recuperado el 2 de agosto de 2014, de https://www.dane.gov.co/files/ investigaciones/condiciones_vida/pobreza/Magdalena_Pobreza_2013.pdf

DANE - Censo General 2005. (2005). Necesidades Básicas Insatisfechas. Recuperado de https://www.dane.gov.co/files/investigaciones/boletines/censo/ Bol_nbi_censo_2005.pdf.

DANE - Censo General 2005. (2005). Perfil Guamal-Magdalena. Recuperado de https://www.dane.gov.co/files/censo2005/perfiles/magdalena/guamal.pdf.

Departamento Nacional de Planeación. (2015). Diagnoóstico de la pobreza rural en Colombia 2010-2014. Bogota: Departamento Nacional de Planeaión.

Departamento Nacional de Planeación. (2015). Observatorio nacional de familias. Boletín número 2. Colombia, Bogotá.

Ernst, F., \& Haushofer, J. (2014). The psychology of poverty: Evidence from 43 countries. Science - siencemag.org, 344, 862-867.

Escalada, M., \& Fernández-Soto, S. (2004). El diagnóstico social: proceso de conocimiento e intervención profesional. Buenos Aires: Espacio. 
Feres, J., \& Mancero, X. (2001). Enfoques para la medición de la pobreza. Breve revisión de la literatura. Santiago de Chile: Cepal - Eclac.

Foster, V., \& Yepes, T. (2006, agosto 30). Is Cost Recovery a Feasible Objective for Water and Electricity? Recuperado de The World Bank .

Giraldo, F., Torres, J., \& Bateman, A. (2004). Habitat y desarrollo humano. PNUD - Un habitat.

Gobierno de Colombia. (2011). Programa de Abastecimiento de Agua y Saneamiento en Zonas Rurales de Colombia (CO-L1105). Colombia. Recuperado de http:// idbdocs.iadb.org/wsdocs/getdocument.aspx?docnum=36748358

Index Mundi. (2017, agosto 25). Recuperado de https://www.indexmundi.com/g/g. aspx? $=\operatorname{co} \& v=24 \& l=e s$

Kawachi, I., Kennedy, B., Lochner, K., \& Prothrow, S. (2011, agosto 30). Social capital, income inequality, and mortality. American Public Health Association. Recuperado de http://ajph.aphapublications.org/doi/abs/10.2105/AJPH.87.9.1491

La Porta, R., Lopez de Silanes, F., Sheleifer, A., \& Vishhny, R. (1997). Trust in large organizatión. American Review Paper and Proceeding, 2(87), 333-338.

Lentini, M., \& Palero, D. (1997, agosto). Hacinamiento la dimension no visible del deficit habitacional. Boletín INVI - Chile, 12(31), 23-32.

Martínez, E. (2002). Aporofobia. En J. Conill. Glosario para una sociedad intercultural (pp. 17-23). Valencia: Bancaja.

Martínez, R. M. (2015, julio 7). Inspectora de Polícia. (R. Mejía, \& N. Gómez, entrevistadores)

Ministerio de Ambiente, Vivienda y Desarrollo Territorial. (2004). Metodología para el análisis de tensiones, interacciones y balances en dinámicas y desarrollos poblacionales ambientales, sociales y económicos. Bogotá: Panamericana Formas e Impresos.

Ministerio de Salud y Proteccion Social. (2013). Envejecimiento demográfico en Colombia 1951-2020. Dinámica demográfica y estructuras poblacionales. Bogota: Imprenta Nacional de Colombia.

Observatorio Nacional de Paz. (2010). Dinámicas recientes del conflicto armado en el departamento del Magdalena. Recuperado el 30 de julio de 2014, de http://www.observapaz.org/index.php?option=com_docman\&task=doc download\&gid=15\&Itemid $=49$.

Pérez, M., \& Pérez, E. (2002). El sector rural en Colombia y su crisis actual. Cuadernos de Desarrollo Rural, 35-38. 
Perfetti, J. (2009). Crisis y pobreza rural en América Latina y Colombia. Programa Dinamicas Territoriales. Santiago: Rimisp.

Perry, S. (2010). La pobreza rural en Colombia. Centro Latinoamericano para el Desarrollo Rural. Recuperado de http://www.rimisp.org/wp-content/files mf/1366386291DocumentoDiagnosticoColombia.pdf

Redaccion Salud - Periódico El Espectador. (2016, diciembre 12). La familia colombiana está cambiando. El Espectador, p. 12. Recuperado de http:// www.elespectador.com/noticias/salud/familia-colombiana-esta-cambiandoarticulo-670085-0

Romero, J. (2006). Movilidad social, educación y empleo: los retos de la política económica del departamento del Magdalena. Cartagena de Indias: Banco de la República. Recuperado de http://www.banrep.gov.co/docum/Lectura_finanzas/ pdf/DTSER-74.pdf

Sánchez, E., Ahmed, K., \& Awe, Y. (2006). Prioridades ambientales para la reducción de la pobreza en Colombia. Un análisis ambiental del país para Colombia. Bogotá: Banco Mundial, Mayol Ediciones S.A.

Sen, A. (1992). Sobre conceptos y medidas de pobreza. Comercio Exterior, 42(4), 13. Recuperado de http://www.derechoshumanos.unlp.edu.ar/assets/files/ documentos/sobre-conceptos-y-medidas-de-pobreza.pdf

Sen, A. (1998). Capital humano y capacidad humana. Cuadernos de Economia, 17(29), 67-72.

Zak, P., \& Nack, S. (2001, abril). Trust and Grouth. TheEconomic Journal III, 295321. 